ACCEPted FOR PUblication in the ApJ

Preprint typeset using $\mathrm{LATEX}_{\mathrm{E}}$ style emulateapj v. 4/12/04

\title{
THE EFFECT OF UV/SOFT X-RAY EXCESS EMISSION ON THE WARM ABSORBER PROPERTIES OF ACTIVE GALACTIC NUCLEI - A CASE STUDY OF IRAS 13349+2438.
}

\author{
Sibasish Laha ${ }^{1}$, Gulab C. Dewangan ${ }^{1}$, Susmita Chakravorty ${ }^{2,3}$, Ajit K. Kembhavi ${ }^{1}$ \\ Accepted for publication in the ApJ
}

\begin{abstract}
The UV to X-ray continuum of active galactic nuclei (AGN) is important for maintaining the ionisation and thermal balance of the warm absorbers (WAs). However, the spectra in the sensitive energy range $\sim 13.6-300 \mathrm{eV}$ are unobservable due to Galactic extinction. Moreover, many AGN show soft X-ray excess emission of varying strength in the $0.1-2 \mathrm{keV}$ band whose origin is still highly debated. This soft-excess connects to the UV bump in the unobserved region of $13.6-300 \mathrm{eV}$. Here we investigate the effect of the assumed physical model for the soft-excess on the flux of the unobserved part of the spectrum and its effect on the WA properties. We perform a case study using the XMM-Newton observations of the bright Seyfert 1 galaxy IRAS $13349+2438$ with WA features. The two different physical models for the soft excess: blurred Compton reflection from an ionised disk, and, optically thick thermal Comptonisation of the disk photons, predict different fluxes in the unobserved energy range. However the current X-ray data quality does not allow us to distinguish between them using derived WA parameters. This, in turn, implies that it is difficult to determine the origin of the soft-excess emission using the warm absorber features.
\end{abstract}

Subject headings: Galaxies: Seyfert, quasars: absorption lines, X-rays: individual: IRAS $13349+2438$

\section{INTRODUCTION}

About half of type I active galactic nuclei (AGN) show soft X-ray absorption features due to partially ionised material along our line of sight and intrinsic to the source (Nandra \& Pounds 1994; Reynolds 1997; George et al. 1998; Blustin et al. 2005; Piconcelli et al. 2005) . Such X-ray absorbing clouds first detected by Halpern (1984) using Einstein data, have been named partially ionised absorbers or "warm absorbers". The availability of high resolution grating X-ray spectra with XMM-Newton and Chandra in the last decade improved enormously our knowledge of these discrete warm absorption and warm emission features in AGN spectra.

The warm absorber (hereafter WA) clouds give rise to narrow absorption lines and edges in the spectrum, from various ionisation stages of several elements (see e.g., Kaastra et al. 2000; Kaspi et al. 2000; Blustin et al. 2005). Absorption features from $\mathrm{H}$-like and He-like ions of $\mathrm{C}, \mathrm{N}, \mathrm{O}, \mathrm{Ne}$ and lower ionisation states of $\mathrm{Fe}$ (including the unresolved transition array (UTA) at $\sim 0.7 \mathrm{keV}$ ) are most prominent in the soft X-rays. These lines and edges are sensitive to photons in the energy range of $13.6 \mathrm{eV}-2 \mathrm{keV}$, and the ionisation structure of the WA clouds depends on the shape and strength of the AGN continuum in that energy range. As such, these lines serve as important diagnostics of the ionisation structure and kinematics of the gas, as well as a probe of the continuum shape.

To model these lines and edges in a dataset and determine the ionisation phase (via an ionization parameter $\xi$ ) and column density $\mathrm{N}_{\mathrm{H}}$ of the cloud, there are a few photo-ionisation codes in vogue, for example, CLOUDY

\footnotetext{
1 Inter University Centre for Astronomy and Astrophysics; email: laha@iucaa.ernet.in ; gulabd@iucaa.ernet.in

2 Harvard University, Department of Astronomy

3 Harvard Smithsonian centre for Astrophysics, 60 Garden street, Cambridge, MA 02138, USA
}

(Ferland et al. 1998), XSTAR (Kallman et al. 2004), etc. These codes require as inputs the ionizing continuum from the source, cloud density and cloud metallicity, among other parameters, to generate a grid in $\xi$ and $\mathrm{N}_{\mathrm{H}}$. The ionizing continuum plays a very important role in determining the parameters of the cloud.

However, it is not always easy to have an accurate estimate of the ionizing continuum as emitted by the central engine and as seen by the WA. Due to Galactic neutral absorption a portion of the ionizing continuum $(13.6-300 \mathrm{eV})$ becomes unobservable to us. This is the region where the two most important parts of an AGN spectral energy distribution (SED), the Big Blue Bump (BBB) and the soft X-ray excess (SE) join. The BBB (peaking at $\sim 1-30 \mathrm{eV}$ ) is believed to have its origin in the accretion disk, as thermal multi-temperature blackbody emission (Shakura \& Sunyaev 1973). The SE, on the other hand, is excess emission at energies $<2 \mathrm{keV}$ over a powerlaw extending to high energies. Till date there has been no consensus on the physical origin of the SE and it can be well described by many prevalent models such as single or multiple blackbodies, high temperature diskblackbody, optically thick thermal Comptonization, blurred reflection from partially ionized disk etc (see e.g., Ross \& Fabian 2005; Done et al. 2012). The different models when used to describe the SE can predict different fluxes when extrapolated to the unobserved portion of the SED; the photons from this part of the spectrum are particularly important for warm absorber clouds. So a physical description of the soft-excess becomes imperative to describe the broad band SED. Moreover, the energy range of the soft-excess $(0.3-2 \mathrm{keV})$ is where the WA features are mainly found. Hence the properties of the WA are likely to depend on the way the SE is modeled.

Nicastro et al. (1999) have studied the properties of the transmitted spectra of a gas illuminated by a flat and a steep X-ray spectrum. They found that differ- 
ent X-ray continua produce distinctly different ionisation structure in a cloud resulting in different absorption features in the energy range $0.1-2 \mathrm{keV}$. Mehdipour et al. (2012) have investigated the effect of the uncertainties in the construction of the SED of the Seyfert 1.8 galaxy ESO 113-G010 on its WA, as it is intrinsically obscured. The uncertainties in the IR and the UV parts of the SED were tested and were found to affect the thermal stability of each phase of the detected warm absorbers. Lee et al. (2013) have studied the warm absorbers of the source IRAS $13349+2438$ using Chandra data. They have found that the presence of the UV bump in the SED creates an increased number of stable phases in the Stability-curves and thereby favor a continous distribution of ionisation states in pressure equilibrium.

In this paper we use the different physical models describing the observed X-ray and the UV data and predict the fluxes in the unobserved part of the continuum $(13.6-300 \mathrm{eV})$. Further we investigate the effect of these different continua on the WA properties, using a case study of an XMM-Newton observation of a bright Seyfert 1 galaxy IRAS $13349+2438$, known to show strong WA features (Sako et al. 2001). We also investigate how the different shapes of the UV and soft $\mathrm{X}$-ray continua affect the WA properties of the source. We further carry out an extensive stability curve analysis for the different SEDs.

The paper is organised as follows. Section 2 deals with the different ionizing continua and their effect on the X-ray spectrum. Section 3 deals with the case study of IRAS $13349+2438$ and describes X-ray observation, modeling of continuum and WA absorbers. This section also describes the construction of an appropriate ionizing continuum for IRAS $13349+2438$ and also investigates the effect of different ionizing continua on the observed WA properties and the stability curve analysis. Section 4 discusses the results followed by conclusions in Section 5. Throughout this work we have used the cosmological parameters $\mathrm{H}_{\mathrm{o}}=71 \mathrm{~km} \mathrm{~s}^{-1} \mathrm{Mpc}^{-1}, \Omega_{m}=0.27, \Omega_{\Lambda}=$ 0.73 to calculate distance.

\section{IONIZING CONTINUUM AND THE WARM ABSORBERS}

The level of ionisation in a warm absorber cloud can be characterised by the ionisation parameter $\xi=L / n r^{2}$, where $L$ is the ionizing luminosity between 1 and 1000 Ryd, $n$ is the hydrogen number density, and $r$ distance between the ionizing source and the illuminated face of the cloud (Tarter et al. 1969). This parameter is defined for hydrogen at the cloud surface facing the ionising radiation. The ionisation structure of the cloud on the other hand, determined by the relative ionic abundances in the photo-ionised gas, depends on the shape of the incident spectrum. Clouds having same ionisation parameter $\xi$ can show different absorption features when illuminated by different SEDs. Nicastro et al. (1999) have found that the steep sloped NLSy1s lack the presence of strong absorption edges of Oxygen and other elements whereas the flat sloped Seyfert 1 galaxies show strong presence of $\mathrm{K} \alpha$ and $\mathrm{K} \beta$ resonance absorption lines from $\mathrm{H}$-like and $\mathrm{He}-$ like ions of $\mathrm{C}, \mathrm{O}, \mathrm{Ne}$, etc. The continuum strength in the soft X-rays therefore play a crucial role in determining the nature of ionic structure of the WA cloud. This has important implication for the extent of ionization in WA clouds, as the photoelectric absorption cross-sections for various elements are generally higher at lower energies above their K-edges. Thus, a continuum with strong extreme ultra-violet and soft X-ray excess emission will produce more ionization compared to a flat continuum with strong hard X-rays. To demonstrate these effects, we have created warm absorber models for four different ionizing continua with varying UV and soft X-ray excess emission. The choice of these continua are driven by the case study we perform on the source IRAS13349+2438 in later sections. The four ionizing continua are as follows.

1. NLSy1 continuum - This continuum is typical of Seyfert 1 galaxies and consists of an X-ray powerlaw (photon index $\Gamma \sim 2$ ), soft X-ray excess below $2 \mathrm{keV}$ described as a blackbody $\left(k T_{B B} \sim 85 \mathrm{eV}\right)$, and a multicolor accretion disk blackbody (BBB) characterised by an inner disk boundary temperature $k T_{i n} \sim 4 \mathrm{eV}$. This is the UV-X-ray continuum obtained from XMM-Newton observation of IRAS $13349+2438$ (see Sect. 3.3) and the BBB as characterised by Lee et al. (2013) (see Sect. 3.5).

\section{NLSy1 continuum without the BBB.}

3. NLSy1 continuum without the SE.

\section{NLSy1 continuum without the BBB and the SE.}

The continua 2, 3 and 4 are generated to investigate the effect of various parts of the ionising continuum on the warm absorber clouds.

The above four ionising continua were used to create WA models using the photoionisation code CLOUDY (version 08.00, see Ferland et al. (1998) for a description of CLOUDY), which uses an extensive atomic database to predict the absorption and emission spectrum through and from a cloud. The clouds are assumed to have a uniform spherical distribution around the central source and are photoionised by the source. The geometry of the cloud is spherical but we approximate it to a plane parallel slab by making the distance of the cloud from the central source very large compared to the thickness of the cloud. CLOUDY performs the simulations by dividing a cloud into thin concentric shells referred to as zones. The thickness of the zones are chosen small enough for the physical conditions across them to be nearly constant. For each zone the simulations are carried out by simultaneously solving the equations that account for ionisation and thermal balance. The model predicts the absorption and emission from such clouds in thermal and ionisation equillibrium. Following Porter et al. (2006), we created WA models for each of the four continua described above.

To see how the warm absorption features of these four WA models affect a spectrum, we have generated a powerlaw spectrum with $\Gamma=2$ in the energy range $0.1-10 \mathrm{keV}$ modified by the four WA models with the same column $\mathrm{N}_{\mathrm{H}}=10^{22} \mathrm{~cm}^{-2}$ and the same ionization parameter $\xi=10 \mathrm{erg} \mathrm{cm} \mathrm{s}^{-1}$. This was done using the $X S P E C$ (Arnaud 1996) spectral fitting package, where we have used a dummy response matrix to generate the fake data. Figure 1 left panel shows the full NLSy1 ionizing continuum in solid black along with the three different constituents of the ionising continuum, the BBB, the SE and the powerlaw with a cut-off. The right panel of Fig. 1 shows the absorption features of the warm 

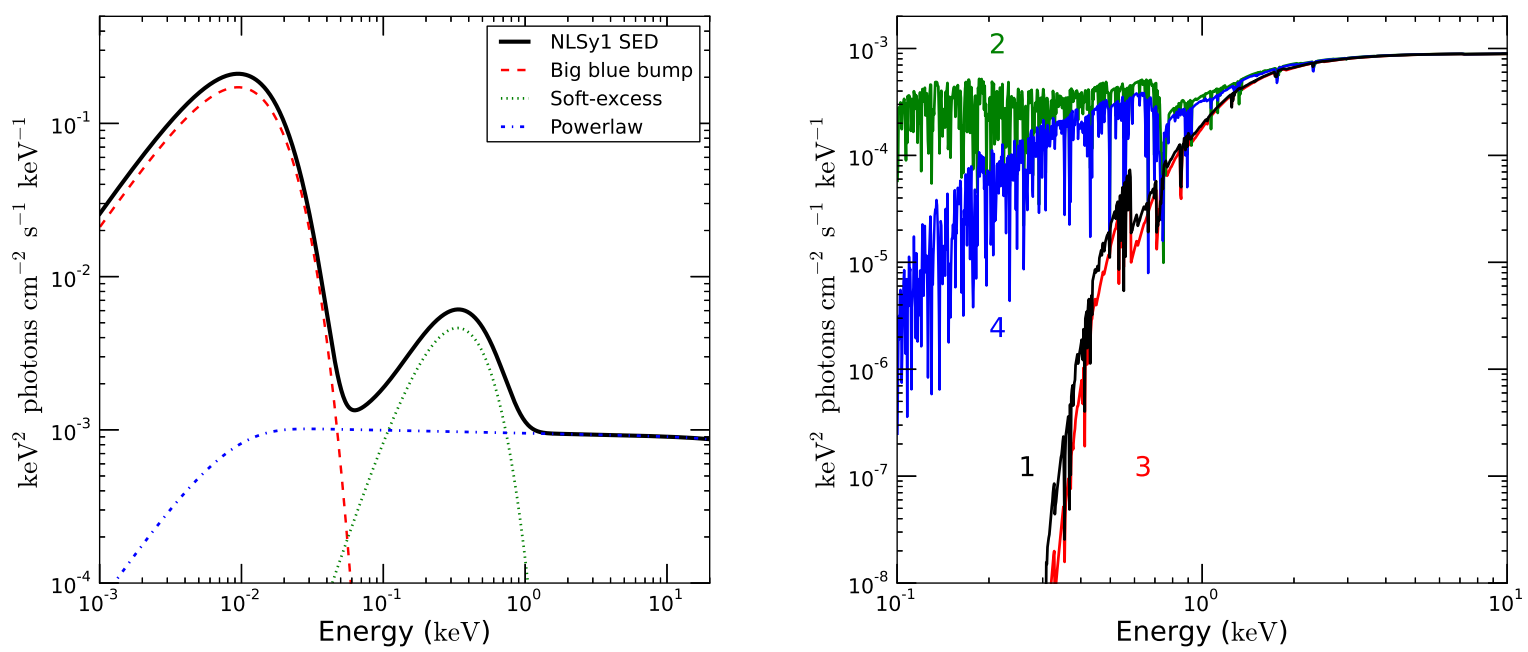

FIG. 1.- LEFT: The black solid line is the NLSy1 ionising continuum as defined in section 2. The red dashed line, the green dotted line and the blue dashed-dotted line shows the three individual components of the continuum, the BBB, the SE and the powerlaw with a cut-off respectively. RIGHT: The absorption features of a warm absorber cloud illuminated four ionising continua as described in section 2 for the same ionisation parameter $\left(\xi=10 \mathrm{erg} \mathrm{cm} \mathrm{s}^{-1}\right)$ and column density $\left(\mathrm{N}_{\mathrm{H}}=10^{22} \mathrm{~cm}^{-2}\right)$ in the energy band of $0.3-10 \mathrm{keV}$. The curves 1,2 , 3 , and 4 denote the absorption from WA clouds illuminated by the four ionising continua, NLSy1, NLSY1 without BBB, NLSy1 without the SE, NLSy1 without the SE as well as the BBB.

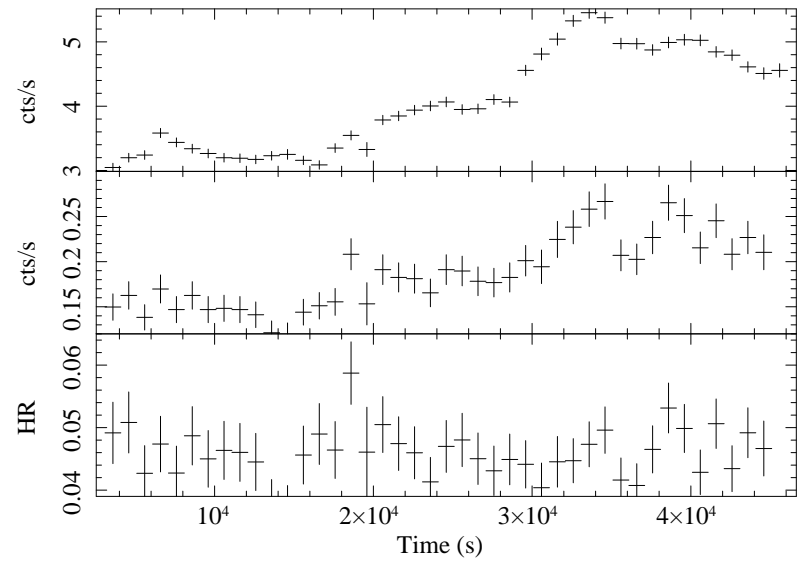

FIG. 2.- The top and the middle panels are $0.2-2 \mathrm{keV}$ and $2-10 \mathrm{keV}$ background subtracted lightcurves respectively of the source IRAS $13349+2438$, for the EPIC-pn data of the $X M M$ Newton observation done in 2000 . The bottom panel is the hardness ratio plot, which is seen to be constant during the observation. Last $15 \mathrm{ks}$ of the observation were not used for spectral analysis due to high particle background flaring.

absorber clouds corresponding to the four SEDs as described above. We see that ionizing continua with different shapes can produce clouds with different absorption features for the same ionization parameter.

This is physically understandable. For example, a continuum with a strong BBB will remove most of the electrons from the atoms in the cloud capable of absorbing in the UV. On the other hand, a continuum with strong SE will remove all the electrons capable of absorbing in the soft X-rays from their shells. So a cloud illuminated by a strong SE is almost 'transparent' in the soft X-rays as it has no further electrons left to absorb the X-rays, as we find in curve 2 in the right panel of Fig. 1. To demonstrate these effects, we have performed a case study of the WA properties of a bright NLSy1 IRAS $13349+2438$ and discuss the effect of different parts of the ionising continuum on the WA properties.

\section{A CASE STUDY OF IRAS $13349+2438$}

IRAS $13349+2438$ is a nearby $(\mathrm{z}=0.107)$ bright radio quiet NLSy1 with a high bolometric luminosity $(\geq$ $10^{46} \mathrm{erg} \mathrm{s}^{-1}$ ). Previous X-ray studies have found the presence of strong WA, SE and a steep powerlaw spectrum with $\Gamma \sim 2.2$ (Longinotti et al. 2003). This is the source in which the Fe unresolved transition array (UTA) absorption features were detected by Sako et al. (2001) for the first time. A multiwavelength study of this source by Lee et al. (2013) has revealed the presence of multiple components of UV and X-ray warm absorbers. IRAS $13349+2438$ has been observed by XMM-Newton on two ocassions in 2000 and once in 2006. In the first observation in 2000 , the X-ray spectrum showed the presence of a strong soft-excess and WA features (Sako et al. 2001). Figure 2 shows the hardness ratio along with the lightcurves in the soft and the hard band. We find that the spectral shape has not varied during the observation. For these reasons we have chosen the source for our study.

\subsection{Observation and data reduction}

We have used the archival XMM-Newton data (id:0096010101) obtained from the observation on 200006-20 for a total exposure of $45 \mathrm{ks}$. The EPIC-pn and MOS cameras were operated in the small window mode using the thin filter. The data were processed using SAS version 12 and the latest calibration database, as available on $3^{\text {rd }}$ March 2012.

The EPIC data were filtered using the standard filtering criterion as well as for particle background which resulted in a net EPIC-pn exposure of $\sim 30$ ks in the EPICpn data which is similar to that obtained by Longinotti et al. (2003). We checked the photon pile-up using the 
SAS task epatplot and found that there was no noticeable pile-up in either EPIC-pn or MOS data. We quote results based on EPIC-pn data due to its higher signal-to-noise compared to the MOS data. We have used the good Xray events $(\mathrm{FLAG}=0$, pattern $=0)$. To extract the source spectrum we used a circular region of 45 arcsec, centred on the centroid of the source. We extracted the background spectrum from appropriate nearby circular regions free of sources. We created the ancillary response file (ARF) and the redistribution matrix file (RMF) using the SAS tasks arfgen and rmfgen. We reprocessed the RGS data using the SAS task rgsproc and the OM data using the SAS task omichain. The OM camera simultaneously observed IRAS $13349+2438$ in the UVW2 filter along with EPIC and RGS cameras. We have obtained the source flux at $2120 \AA$ from the data and corrected it for Galactic extinction following Schlafly \& Finkbeiner (2011) and Schlegel et al. (1998). We calculated the monochromatic flux at $2120 \AA$ from the AGN to be $3.4 \times 10^{-15} \mathrm{erg} \mathrm{cm}^{-2} \mathrm{~s}^{-1} \AA^{-1}$.

\subsection{X-ray spectral analysis}

We begin with the spectral analysis of the broadband $(0.3-10 \mathrm{keV})$ EPIC-pn spectral data. The data were grouped with a minimum of 20 counts per energy bin and allowing 5 energy bins per resolution element. This was done using the specgroup tool in the SAS. We used ISIS version 1.6.2-12 (Houck \& Denicola 2000) for our spectral fitting. An absorbed powerlaw fit to the spectrum in the $2-10 \mathrm{keV}$ band yielded a powerlaw slope $\Gamma=1.96_{-0.09}^{+0.08}$ which is similar to that obtained by Longinotti et al. (2003). There is possibly a very weak narrow $\mathrm{Fe} \mathrm{K \alpha}$ line and an Fe $\mathrm{K}$ absorption edge. We fitted the $\mathrm{Fe} \mathrm{K} \alpha$ emission line with a narrow Gaussian and the fit improved by only $\Delta \chi^{2}=-4$ for 2 extra parameters from $\chi^{2} /$ dof $=127 / 125 \sim 1.02$, where dof stands for degrees of freedom. The Fe K edge was fitted using an edge model in ISIS. The fit improved by $\Delta \chi^{2}=-28$ for 2 extra parameters. The best fit edge energy is $7.48_{-0.18}^{+0.12} \mathrm{keV}$, and the maximum optical depth $\tau=0.44 \pm 0.22$, similar to that found by Longinotti et al. (2003). The statistical improvement according to an F-test upon addition of this component is $>99.9 \%$. However the best fit $\chi^{2} /$ dof $=99 / 123$ suggests that the data may be overmodeled in this energy band.

We extrapolated the model to the softer part of the spectrum and found a prominent soft excess which was well described by a blackbody with a best fit temperature of $\mathrm{kT}_{\mathrm{BB}}=85 \pm 2 \mathrm{eV}$ in the energy range $0.3-10 \mathrm{keV}$, which is again similar to that found by Longinotti et al. (2003). In Figure 3, the left panel shows clear residuals of absorption features in the soft X-ray band, which are mainly the signatures of absorption features. We used CLOUDY models to fit these absorption features.

\subsection{Constraining the broad band $U V$ to $X$-ray continuum}

The WA features in the X-rays consist of a number of absorption lines and edges of varying strengths and are usually unresolved and blended. In such cases, inferring $\mathrm{X}$-ray continua from moderate resolution X-ray spectra requires appropriate physical models of the WA cloud. However, creating such a model requires the knowledge of the ionizing continuum seen by the WA. This is somewhat of a circular problem. We determine the continuum in the $0.3-10 \mathrm{keV}$ band in the following way: We first use a generic AGN continuum to generate the CLOUDY WA table models. We use these WA models to fit the $0.3-10 \mathrm{keV}$ EPIC-pn and the RGS data jointly to the $\mathrm{X}$-ray continuum (Section 3.4). The BBB is then derived following Lee et al. (2013) (section 3.5). Finally we extrapolate the BBB and the X-ray continuum to the unobserved energy range of $13.6-300 \mathrm{eV}$ and obtain the appropriate SED for the source. This SED is then used to create the WA table model and we obtain the best fit WA and continuum parameters from the joint fit of EPIC-pn and RGS data.

\subsection{The X-ray continuum}

In the first step we use the AGN continuum given by Korista et al. (1997) to generate the warm absorber table models in CLOUDY. The Kirk Korista continuum is given by

$$
f_{\nu}=\nu^{\alpha_{u v}} \exp \left(-h \nu / k T_{\mathrm{BBB}}\right) \exp \left(-k T_{\mathrm{IR}} / h \nu\right)+\eta \nu^{(1-\Gamma)},
$$

This consists of a power-law in the $1 \mathrm{eV}-100 \mathrm{keV}$ band, and another steeper power-law in the UV whose upper exponential cutoff is parametrised with a temperature $T_{\mathrm{BBB}}$ and the lower infrared cut off by $T_{\mathrm{IR}}$. We used a typical Seyfert 1 X-ray powerlaw slope of $\Gamma=2$, a UV bump blackbody temperature of $\mathrm{T}_{\mathrm{BBB}}=10^{5} \mathrm{~K}$ peaking at $\sim 10 \mathrm{eV}$, and an $\alpha_{o x}=-1.2$. The UV spectral slope was assumed to be $\alpha_{u v}=-0.5$ (Elvis et al. 1994). The CLOUDY table model was built using the methods described in Porter et al. (2006). We varied $\log \left(\xi / \mathrm{erg} \mathrm{cm} \mathrm{s}^{-1}\right)$ from -2 to 4 and $\log \left(\mathrm{N}_{\mathrm{H}} / \mathrm{cm}^{-2}\right)$ from 19 to 24 and created a multiplicative table model for the warm absorption. The cloud was assumed to have solar metallicity. A hydrogen density of $n_{H} \sim 10^{9} \mathrm{~cm}^{-3}$ was assumed as the properties of the WA clouds are practically volume density independant in the range $\sim 10^{2}-10^{12} \mathrm{~cm}^{-3}$ (Netzer 1996). The table model was subsequently imported to the ISIS package and was used to obtain the best fit $0.3-10 \mathrm{keV}$ continuum parameters by carrying out a joint fit with the EPIC-pn and the RGS data. The best fit continuum parameters are: powerlaw slope $\Gamma=2$, and bbody $k T_{e}=85 \mathrm{eV}$. Two ionisation states of WA were detected with best fit parameters: for the lower ionisation state $\log \xi=1.50$, $N_{\mathrm{H}}^{\mathrm{WA}}=2 \times 10^{21} \mathrm{~cm}^{-2}$, and for the higher ionisation state, $\log \xi=2.25, N_{\mathrm{H}}^{\mathrm{WA}}=2 \times 10^{21} \mathrm{~cm}^{-2}$. We report the best fit continuum and the WA parameters in Table 1 .

\subsection{Characterising the Big Blue Bump}

The BBB in AGN is primarily thought to arise from an optically thick but geometrically thin accretion disk following Shakura \& Sunyaev (1973). We used the diskbb model (Makishima et al. 1986) in ISIS to define the shape of the $\mathrm{BBB}$, which requires the blackhole mass and distance to the source for the normalisation to be determined. We have characterised the UV bump following Lee et al. (2013). They have derived the diskbb parameters for the same source from the optical-UV data points 

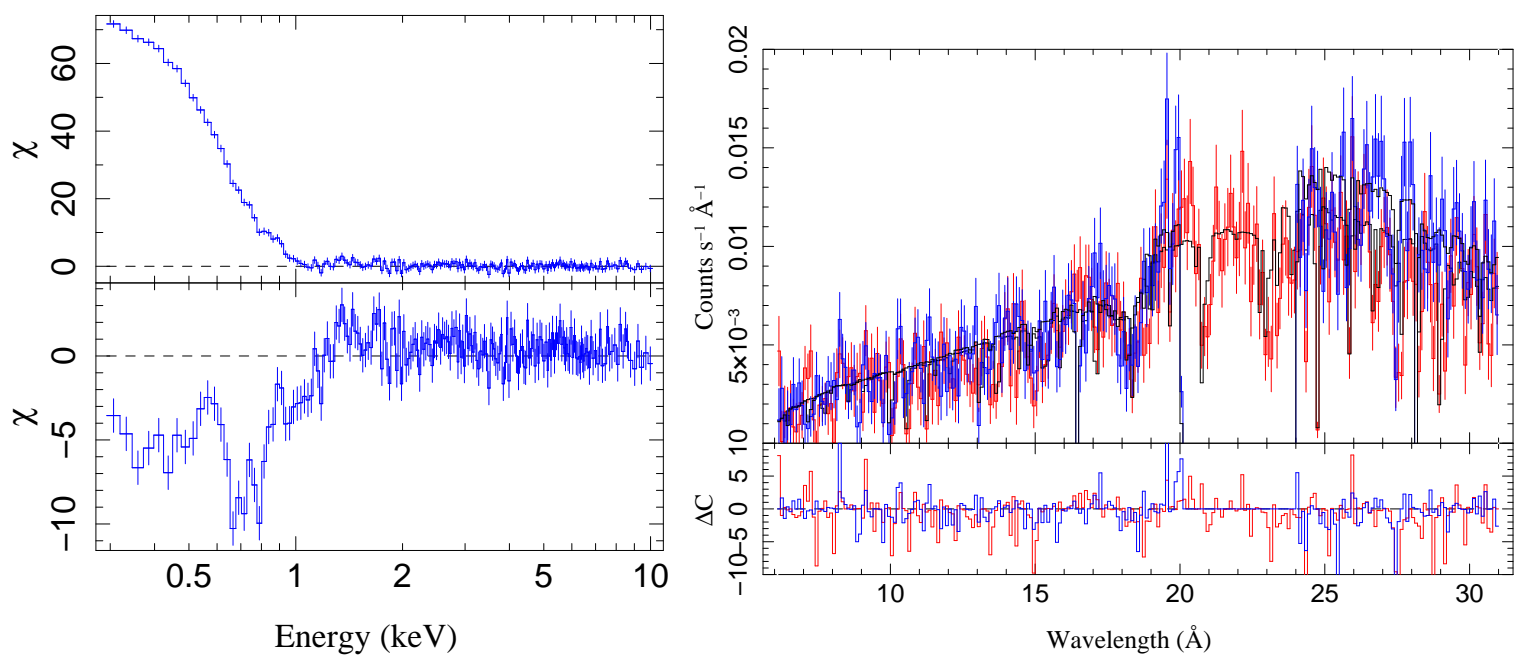

FIG. 3.- LEFT : EPIC-pn data residuals expressed as $\chi=($ data - model $) / \sigma$, where $\sigma$ denotes the statistical errors on the data. The upper panel shows the prominent soft-excess emission over an absorbed powerlaw fit in the $2-10 \mathrm{keV}$ range. The lower panel shows the presence of absorption features after we add a blackbody of temperature $\mathrm{kT}_{\mathrm{BB}}=0.085 \mathrm{keV}$ to the previous fit. RIGHT : The RGS1 and RGS2 datasets in blue and red respectively, with the best fit model in black when fitted simultaneously with EPIC-pn data using the warm absorbers developed using the appropriate continuum (SE modeled with bbody).

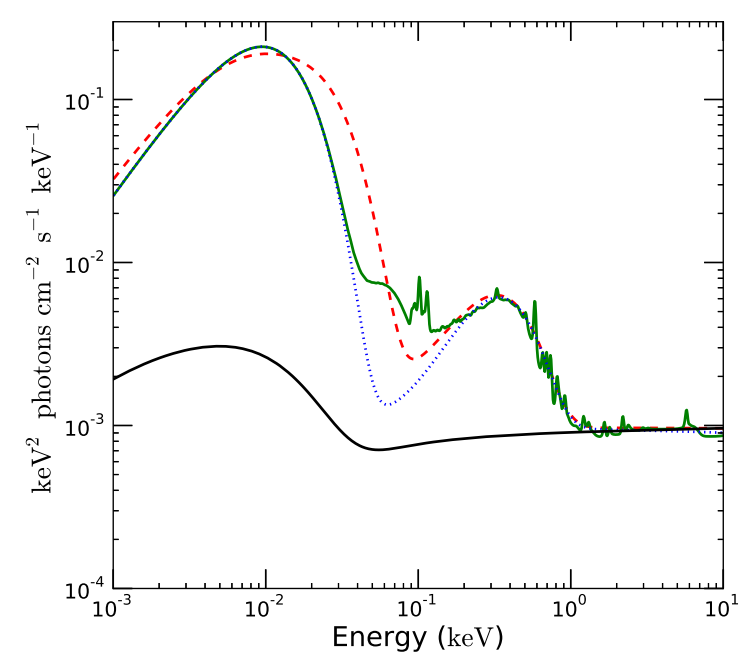

FIG. 4.- The different realistic ionizing continua and their comparison with the generic Kirk Korista continuum shown by black solid curve. The dotted blue, dashed red, and solid green curves stand for SEDs where the soft excess is modeled by bbody, optxagnf and reflionx models respectively. See Section 3.7 for a discussion.

obtained using Hubble Space Telescope (HST) after correcting for the intrinsic galactic reddening. The mass of the central massive blackhole of IRAS $13349+2438$ galaxy is estimated to be $\mathrm{M}_{\mathrm{BH}}=10^{8.75} \mathrm{M}_{\odot}$, an accretion efficiency of $20 \%$ with respect to the Eddington rate and an inner radius of $10 \mathrm{R}_{\mathrm{s}}$ (Schwarzschild radius). The normalisation of the diskbb is obtained from these parameters and the luminosity distance of the source which is $483 \mathrm{Mpc}$. Although the UV data used by Lee et al. (2013) is non simultaneous to the XMM-Newton observation we are working with, the fluxes by them are similar to those obtained by us using the OM UVW1 filter.

\subsection{The UV to Xray continuum and the Warm Absorber properties}

We now develop a model ionising continuum as emitted by the central active engine and as seen by the WA for the UV to X-ray energy band $1 \mathrm{eV}-10 \mathrm{keV}$. For the $0.3-10 \mathrm{keV}$ band continuum, the best fit continuum model parameters obtained from the broad band EPICpn and RGS data in Section 3.3 is used, while the BBB is used as described in Section 3.5. In the unobserved energy range of $\sim 13.6-300 \mathrm{eV}$ the $\mathrm{BBB}$ meets the extrapolated $0.3-10 \mathrm{keV}$ continuum. Thus using the UV and X-ray observations we have derived an appropriate continuum which is most likely seen by the WA clouds in IRAS $13349+2438$. Fig. 4 shows this continuum as a blue dotted line.

This ionizing continuum (hereafter 'appropriate' continuum) is used to generate WA model in CLOUDY which is then used to fit the EPIC-pn and RGS data simultaneously. The best fit WA parameters obtained are: $\log \xi \sim 1.75, N_{\mathrm{H}}^{\mathrm{WA}} \sim 2 \times 10^{21} \mathrm{~cm}^{-2}$ for the lower ionisation state, and $\log \xi \sim 3.25, N_{\mathrm{H}}^{\mathrm{WA}} \sim 2 \times 10^{21} \mathrm{~cm}^{-2}$ for the higher ionisation state (see Table 1 for details). We note that both the ionisation states are different from those obtained using the WA model developed using the Kirk Korista ionizing continuum, although the continuum parameters are similar.

\subsection{Realistic ionising continuum and physical models for the Soft-excess}

In our attempt to create an appropriate ionizing continuum we have used a blackbody to describe the softexcess, which however is only a phenomenological description and the resulting continuum may not be realistic. In the absence of observations in the $13.6-300 \mathrm{eV}$, the best solution to derive the realistic continuum is to use physical models for the SE and the BBB. Though the nature of the SE is not clearly understood, it can be well described by two physical models (1) optically thick thermal Comptonisation (optxagnf, in ISIS nota- 
tion) and (2) blurred reflection from partially ionised accretion disk (reflionx, in ISIS notation ).

The optxagnf model proposed by Done et al. (2012) to model the soft-excess involves Comptonisation of disk seed photons from a complex geometry. The AGN spectral energy distributions in the UV to X-rays can be phenomenologically described by three main emission components: (1) the disk emission in the UV, (2) The soft Xray excess emission from an optically thick, low temperature thermal Comptonizing plasma, and (3) and power law emission above $2 \mathrm{keV}$ from an optically thin, high temperature Comptonizing plasma. In the optxagnf model these three main components of the spectra are combined together assuming that they are all ultimately powered by gravitational energy released in the accretion process. The optxagnf model therefore simultaneously describes the UV as well as the X-ray spectra. However, we have only one data point in the UV from the OM UVW2 filter for the given observation which is not sufficient to constrain the model parameters. We created a set of fake datasets (in ISIS) in the UV $(1 \mathrm{eV}-10 \mathrm{eV})$ using the measured BBB described by Lee et al. (2013) (see Section 3.5). We assumed a typical $5 \%$ systematic errors on the UV data points. This UV data are simultaneously fitted with the EPIC-pn data to obtain the broadband UV to X-ray SED (see Figure 4, red dashed line). The model used is wabs $\times$ WA $1 \times$ WA $2 \times$ edge $\times($ optxagnf + Gaussian $)$ in ISIS notation. The WA models used are those developed in the Section 3.6. This continuum is now used to generate the WA model corresponding to the newly derived optxagnf SED. We used this WA model to fit only the X-ray data $(0.3-10 \mathrm{keV})$ with the model mentioned above and obtained the best fit WA and continuum parameters. The best fit parameter values of optxagnf model are $\log \left(\mathrm{L} / \mathrm{L}_{\mathrm{Edd}}\right) \sim-0.76, \Gamma \sim 1.99$ and the temperature of the thermal optically thick Comptonizing electrons responsible for SE are $k T_{e}=101 \mathrm{eV}$. The best fit WA parameters obtained are $\log \xi=1.95$, $N_{\mathrm{H}}^{\mathrm{WA}}=2 \times 10^{21} \mathrm{~cm}^{-2}$ for the lower ionisation state, and $\log \xi=3.52, N_{\mathrm{H}}^{\mathrm{WA}}=2 \times 10^{21} \mathrm{~cm}^{-2}$ for the higher ionisation state (see Table 3 and 4 for details). We note here that the normalization of optxagnf was frozen to one, since the flux is completely calculated by the four parameters: the blackhole mass $\mathrm{M}_{\mathrm{BH}}$, the spin of the blackhole, the mass accretion rate $L / L_{E d d}$, and the luminosity distance of the source $D_{L}$.

The reflionx model (Ross \& Fabian 2005) describes the soft-excess as Compton reflection of hard X-ray photons from an ionised disk. It assumes a semi-infinite slab of optically thick cold gas of constant density, illuminated by a powerlaw producing a reflection component including the fluoresence lines from the ionised species in the gas. The XSPEC model kdblur was convolved with the reflection model to account for the Doppler and gravitational effects. The $0.3-10 \mathrm{keV} \mathrm{X}$-ray spectrum was initially fitted with a model wabs $\times$ WA $1 \times$ WA $2 \times$ (powerlaw + kdblur(reflionx)) in ISIS notation. The WA models used are those created using the appropriate continuum (Sec. 3.6). The above model did not describe the data well $\left(\chi^{2} /\right.$ dof $\left.=305 / 177\right)$. There were still some narrow discrete residuals in the soft X-rays. We added one more reflection component which is unblurred and is as- sumed to arise from a distant reflector. The fit statistic improved to $\chi^{2} /$ dof $=214 / 174$. We further tested the fit using more complex reflection models. We added one more blurred reflection component which arises from the same disk as the first one but lies on the outer side. We tied the outer radius of the first reflection component to the inner radius of the second component and let them free to vary. Since they arise from the same disk, we also assume that they have the same Fe abundance and same inclination angle with respect to the line of sight and we tie those two parameters as well. On addition of this component the fit improved to $\chi^{2} /$ dof $=201 / 171$ which is acceptable. No further reflection component was necessary. This X-ray continuum along with the BBB in the UV (described in Sec. 3.5) were used to create the broad band SED which was used to generate the WA table models corresponding to the reflionx model. We used those WA models to fit the X-ray data. The EPICpn and the RGS data were simultaneously fitted with wabs $\times$ WA $1 \times$ WA $2 \times($ powerlaw + kdblur $($ reflionx $)+$ $\operatorname{kdblur}(2)(\operatorname{reflionx}(2))+$ reflionx $)$. Table 4 column 3 enumerates the best fit parameters obtained in the fit. Fig. 4 shows the three different ionizing continua derived when we used the different models to describe the soft-excess. We find that the WA parameters obtained for different soft excess models used, are similar within errors (see Table 3). Hereafter these two continua will be referred to as the 'realistic continua'.

\subsection{Effect of various continuum components on the warm absorber models}

We constructed three new ionizing continua to investigate the individual effects of the SE and the BBB by removing these components alternately from the appropriate ionizing continuum generated earlier (Sec. 3.6). First we removed the diskbb component to get the NLSy1 continuum without the BBB, and created the WA model. Next we created another WA model using the NLSy1 continuum without the SE. Finally we removed both the $\mathrm{SE}$ and the BBB from the NLSy1 continuum and created a WA model. The continua used are shown in Fig 1. We used these WA models based on different ionising continua to fit the absorption features in the EPIC-pn and RGS data. For each case we obtained a lower and higher ionisation state with different ionisation parameter. These are listed in Table 2.

\subsection{Stability curve analysis}

In the WA temperature range $10^{4}-10^{6.5} \mathrm{~K}$, photoionization is the main heating agent. Radiative recombination and line emission are dominantly responsible for cooling the gas. The WA is assumed to be in ionization and thermal equilibrium where the above physical processes balance each other.

The stability curve, which is a plot of the temperature $T$ against the pressure $\xi / T$, is an effective tool that is often used to study the stability properties of warm absorbers. Every point on this curve represents a possible equilibrium state of the gas (Krolik et al. 1981; Krolik \& Kriss 1995; Reynolds \& Fabian 1995; Komossa \& Meerschweinchen 2000; Komossa \& Mathur 2001; Krongold et al. 2003; Netzer et al. 2003; Krongold et al. 2005a,b; Chakravorty et al. 2009, 2012). A point on a portion of the curve with positive slope corresponds to a state of 



FIG. 5.- LEFT: The stability curves for the Kirk Korista continuum (solid black line) and for three other SEDs described in Sections 3.6 and 3.7 where the SE is described by three different models (bbody, optxagnf, reflionx). The filled circles are the two components of the best fit WA models for the respective continua. The WA components for the Kirk Korista continuum lie on the unstable part of the stability curve, but the WA components due to the realsitic continua lie on stable parts. RIGHT: The stability curves for the test continua where certain components of an AGN ionizing continuum have been switched off, as discussed in Section 3.8 and Table 2. The stability curve for the ionizing continuum where the BBB is switched off, SE is switched off, both SE and BBB are switched off are represented by the dashed green curve, dotted blue curve and dashed-dotted red curve. The corresponding best fit WA models are denoted by red points.

stable thermal equilibrium because any small change in temperature will be countered by the physical processes to leave its physical conditions unchanged. However, if an absorber lies at a position on the curve with negative slope, then any small change in temperature will result in runaway heating or cooling until the properties of the gas adjust to reach stable configuration at higher or lower temperatures.

The stability curve for a given continuum is generated using CLOUDY by stepping through different values of the ionisation parameter and calculating the corresponding equilibrium temperature of the cloud.

The left panel of Figure 5 shows the stability curves for the Kirk Korista continuum and the three other continua developed for the source (SE modeled with bbody, optxagnf and reflionx). The Kirk-Korista continuum stability curve (solid black line) shows distinct regions of stable phases (positive slopes) separated by intermediate unstable phases (negative slopes). Such a stability curve would predict discrete phases of WA which may be in pressure equilibrium with each other. This is because $\xi / \mathrm{T}$ is essentially the ratio between radiation pressure and gas pressure if the distance from the ionizing source is the same for the different clouds of ionized gas. The solid blue points on the curve show the two components of the WA that were derived from fitting the data. We find that both the components of the WA fall in nearly unstable regions of the stability curve. On the other hand, the curve due to the two realistic continua (optxagnf and reflionx) predict mostly stable gas. Such curves correspond to a continuous distribution of temperature and pressure for the absorbing gas. The WA components predicted by fitting the table models of these ionizing continua (see Table 3) are all thermally stable. Thus, for IRAS13349+2438, the stability curve analysis points to the fact that the realistic ionising continua are the preferred ones for producing stable states of the warm absorbers. In the right panel of Figure 5 we compare the stability curves derived using the appropriate SED for IRAS $13349+2438$ (see Sec. 3.6) with stability curves obtained by dropping the BBB and the SE components respectively from the SED. We have shown in the figure with red circles the WA components predicted by fitting the data with the WA models created using the respective continua. We find that the $\mathrm{BBB}$ as well as the SE components are crucial in establishing a largely stable curve and having the WA components in the stable parts of the curve.

\section{DISCUSSION}

We have shown that ionizing continua with different shapes can result in different ionisation structures for a cloud even if they have the same value for the ionisation parameter $\xi$ (see Fig. 1, see also Nicastro et al. 1999; Reynolds \& Fabian 1995). The $13.6-300 \mathrm{eV}$ range in the UV to X-ray SED of AGN is not accessible to us with our current state of the art instruments. This is the energy range whose photons are important for maintaining the ionisation balance of the WA clouds. Several authors in the past have used different techniques to overcome this difficulty. In many cases, the authors have used a single powerlaw ionising continuum with typical photon index of $\Gamma \sim 1.5-2$ in the UV to X-ray band (e.g., Reynolds \& Fabian 1995; Matt et al. 2011; Lobban et al. 2011; Winter et al. 2012). In some cases a powerlaw connecting the two observed fluxes at $2 \mathrm{keV}$ and $2500 \AA$ characterised by a slope $\alpha_{O X}$ has been used for the unobserved part of the continuum (see e.g., Netzer 1993; Longinotti 
et al. 2008; Nucita et al. 2010). This method ignores the presence of the SE, which we have seen is important in maintaining the ionisation balance of the WA. Some authors have modeled the UV data with a powerlaw and joined the last UV data point, for instance, $2120 \AA$ from the OM telescope, with the lowest available X-ray data point $(\sim 300 \mathrm{eV})$ to create the broad band SED (see for e.g., Gupta et al. 2013; Krongold et al. 2005b; Yaqoob et al. 2003). Dewangan et al. (2007) have treated the soft X-ray excess as disk blackbody emission and used a UV powerlaw to construct the ionising continuum.

To constrain the spectral shape in the unobserved energy range, the best one can do is to use physical models to describe the BBB and the SE using the observed data and extrapolate them into the unobserved range. Lee et al. (2013) have modeled the SE in the Chandra HETG spectrum of IRAS $13349+2438$ with a physical model, nthcomp, which involves Comptonisation of seed photons from an accretion disk by a thermal gas of electrons, and extrapolated the resulting continuum below $100 \mathrm{eV}$ where it meets the BBB in the unobserved energy range. In the present case study of the NLSy1 galaxy IRAS $13349+2438$ using XMM-Newton data we have followed a similar procedure, but we have used two additional physical models to describe the SE - the intrinsic disk thermal Comptonisation (optxagnf) and the blurred reflection from an accretion disk (reflionx). The optxagnf model is similar to nthcomp model for the SE but it also produces the BBB and the hard Xray powerlaw self consistently. We find that these different models when extrapolated to the unobserved energy range predict different fluxes (see Fig. 4). The model optxagnf which simultaneously describes the X-ray and the UV data predicts greater flux in the $10-100 \mathrm{eV}$ range compared to the bbody model. The reflionx model which required a separate diskbb model to characterise the $\mathrm{BBB}$ predicts a greater flux in the energy range $20-100 \mathrm{eV}$. The predicted fluxes in the $13.6-300 \mathrm{eV}$ for the three cases optxagnf, reflionx and bbody are $2.5 \times 10^{-10} \mathrm{erg} \mathrm{cm}^{-2} \mathrm{~s}^{-1}, 1.5 \times 10^{-10} \mathrm{erg} \mathrm{cm}^{-2} \mathrm{~s}^{-1}$, and $1.4 \times 10^{-10} \mathrm{erg} \mathrm{cm}^{-2} \mathrm{~s}^{-1}$, respectively. However the X-ray data we are working with cannot distinguish between the WA models created by the two physical SEDs. We find that the two different models lead to similar best fit WA parameters (see Table 3). The implication of these results is that the observed WA properties cannot be used to distinguish between the currently available physical models for the SE.

The ambiguity in the derived WA ionisation parameters for a source due to the uncertainty in the SED means that we cannot determine the 'unique' ionisation parameter for a given cloud for a given dataset. For example, when we use the appropriate SED (described in section 3.6) for the source IRAS $13349+2438$ we obtained the best fit ionisation parameters for the WA clouds $\log \xi \sim 1.75$ and $\log \xi \sim 3.25$, whereas the WA models developed using the SED when the BBB is switched off from the NLSy1 SED yielded completely different best fit WA ionisation parameters $\log \xi \sim 0.99$ and $\log \xi \sim 2.50$. Sako et al. (2001) studied the same observation of the source IRAS $13349+2438$ and obtained two phase WA clouds. After modeling the continuum, they applied the absorption components of individual ions $\mathrm{H}$ and He-like C, N, O and Ne and Fe XVII-XXIV, where each ion was treated as a separate component in the spectral fit. From the observed distribution of the charge states of these ions, they derived the average ionisation parameters using XSTAR: for the lower ionisation state $\log \xi \leq 1.0, N_{\mathrm{H}}^{\mathrm{WA}} \sim 10^{21} \mathrm{~cm}^{-2}$, and outflow velocity is $\mathrm{v} \sim 400 \mathrm{~km} \mathrm{~s}^{-1}$, and for the higher ionisation state $2.0 \leq \log \xi \leq 2.5, N_{\mathrm{H}}^{\mathrm{WA}} \sim 10^{22} \mathrm{~cm}^{-2}$ and outflow velocity is $\mathrm{v} \sim 0 \mathrm{~km} \mathrm{~s}^{-1}$. We found that both ionisation parameters are consistent with those derived by us when we used the WA models based on the appropriate continuum without the BBB (see Table 2). The ionisation parameters obtained by us using the WA model based on realistic continuum are higher than those obtained by Sako et al. (2001), however they are similar to those obtained by Lee et al. (2013). Mehdipour et al. (2012) have found different best fit WA parameters when they used different SEDs to generate the WA models. We found that the outflow velocity and the column density parameters for the WA models generated using different SEDs are statistically similar which is also found by Mehdipour et al. (2012).

The structure of the warm absorber clouds is still elusive. There is as yet no consensus on whether the clouds exist in clumpy discrete phases or as continuos plasma. In two of the high quality X-ray spectra of NGC 3783 (Netzer et al. 2003) and NGC 5548 (Steenbrugge et al. 2003) the data required three ionisation phases. It is not clear if these AGNs really host discrete WA phases as the current data seem to suggest or the number of WA phases will increase with increasing data quality, indicating a continuous distribution of WA clouds. Various studies have however pointed towards a growing consensus on the discrete phases of WA (Blustin et al. 2003; Smith et al. 2008; Ricci et al. 2010; Mehdipour et al. 2010; Ebrero et al. 2011). The stability curves provide a way to distinguish between the two scenarios. For the source IRAS $13349+2438$ we have found that the effect of the $\mathrm{BBB}$ and the SE on the stability curves is to enable stable WA phases over a wide range of $\xi / T$ (see also Reynolds \& Fabian 1995; Fabian et al. 1986). Therefore the continuum might influence the formation of specific phases. Fabian et al. (1986) showed that the two phase discrete WA model for the source Mrk 841 is not valid as the stability curves exhibited more stable regions when they added a SE component to an existing simple powerlaw model in the X-ray continuum. We find that the stability curve generated using the Kirk Korista continuum which has no SE, has discrete stable and unstable phases. The best fit WA parameters for this continuum lie on the unstable portions of the curve. This may point towards a picture where the WA clouds exist in clumpy phases. On the contrary the stability curves generated using the realistic SED for IRAS13349+2438 with the BBB and SE (Fig. 5, left panel) has only stable phases and the best fit WA parameters lie always on stable parts of the curves. This points to a continuos distribution of WA clouds as the available phase space in $\xi / T$ is largely stable. This can impact our understanding of the formation of the WA (Reynolds \& Fabian 1995). Lee et al. (2013) also point out that the presence of a strong UV and SE component creates more stable phases in the stability curve. Holczer et al. (2007) had used only the X-ray continuum for the same source to generate stability curves and found 
unstable phases, which are not present when the SED by Lee et al. (2013) is used. Therefore we find that the stability curves generated using the realistic continua prefer a continuous distribution of WA ionisation states. However, as a caveat we may consider that the detection of two discrete WA components in the stable region of the S-curve may not always imply that there is a continuous distribution of ionization states in the absorber along the line of sight to this target. The S-curves built using the 'realistic' continua shows largely stable portions and allow for a continuos distribution of ionisation states and yet we detect only two discrete states. The fact that we see only 2 components with very different ionization degrees and nothing in between, may argue against the continuous-flow distribution of ionization states.

\section{CONCLUSION}

In this paper we have investigated the effects of the shape of the ionizing continuum on the warm absorber properties. The main results are as follows.

- Ionizing continua with different shapes create different ionisation structures in WA clouds for the same ionisation parameter and column density of the cloud. The best-fit WA parameters obtained using WA models generated with different input ionizing continua for the Seyfert 1 galaxy IRAS $13349+2438$ are different.

- The determination of the accurate shape of the ionising continuum therefore becomes imperative for generating the WA models, which is however not possible due to the Galactic extinction in the range
$13.6-300 \mathrm{eV}$. The only way out is to characterise the BBB and the SE with physical models and extrapolate them in to the unobserved region of the SED.

- We developed realistic continua based on multiwavelength observations which consists of the SE and a powerlaw emission in the X-rays and the $\mathrm{BBB}$ in the UV. We found that the different physical models for SE (blurred Compton reflection and optically thick thermal Comptonisation) predict different fluxes in the unobserved energy range, but the current X-ray data quality does not allow us to distinguish between them using derived WA parameters.

- The extent of stable regions in the stability curves is large for the realistic continuum which possibly indicates a continuous distribution of the WA clouds for this source.

Acknowledgements : This work is based on observations obtained with XMM-Newton, an ESA science mission with instruments and contributions directly funded by ESA Member States and NASA. This research has made use of the NASA/IPAC Extragalactic Database (NED) which is operated by the Jet Propulsion Laboratory, California Institute of Technology, under contract with the National Aeronautics and Space Administration. SL is grateful to CSIR, Government of India for supporting this work. The authors are grateful to the anonymous referee for helpful comments and suggestions.

\section{REFERENCES}

Arnaud, K. A. 1996, in Astronomical Society of the Pacific Conference Series, Vol. 101, Astronomical Data Analysis Software and Systems V, ed. G. H. Jacoby \& J. Barnes, 17

Blustin, A. J., Page, M. J., Fuerst, S. V., Branduardi-Raymont, G., \& Ashton, C. E. 2005, A\&A, 431, 111

Blustin, A. J., et al. 2003, A\&A, 403, 481

Chakravorty, S., Kembhavi, A. K., Elvis, M., \& Ferland, G. 2009 MNRAS, 393, 83

Chakravorty, S., Misra, R., Elvis, M., Kembhavi, A. K., \& Ferland, G. 2012, MNRAS, 422, 637

Dewangan, G. C., Griffiths, R. E., Dasgupta, S., \& Rao, A. R. 2007, ApJ, 671, 1284

Done, C., Davis, S. W., Jin, C., Blaes, O., \& Ward, M. 2012, MNRAS, 420, 1848

Ebrero, J., et al. 2011, A\&A, 534, A40

Elvis, M., et al. 1994, ApJS, 95, 1

Fabian, A. C., Guilbert, P. W., Arnaud, K. A., Shafer, R. A., Tennant, A. F., \& Ward, M. J. 1986, MNRAS, 218, 457

Ferland, G. J., Korista, K. T., Verner, D. A., Ferguson, J. W., Kingdon, J. B., \& Verner, E. M. 1998, PASP, 110, 761

George, I. M., Turner, T. J., Netzer, H., Nandra, K., Mushotzky, R. F., \& Yaqoob, T. 1998, ApJS, 114, 73

Gupta, A., Mathur, S., Krongold, Y., \& Nicastro, F. 2013, ApJ, 768,141

Halpern, J. P. 1984, ApJ, 281, 90

Holczer, T., Behar, E., \& Kaspi, S. 2007, ApJ, 663, 799

Houck, J. C., \& Denicola, L. A. 2000, in Astronomical Society of the Pacific Conference Series, Vol. 216, Astronomical Data Analysis Software and Systems IX, ed. N. Manset, C. Veillet, \& D. Crabtree, 591

Kaastra, J. S., Mewe, R., Liedahl, D. A., Komossa, S., \& Brinkman, A. C. $2000, A \& A, 354, \mathrm{~L} 83$

Kallman, T. R., Palmeri, P., Bautista, M. A., Mendoza, C., \& Krolik, J. H. 2004, ApJS, 155, 675
Kaspi, S., Brandt, W. N., Netzer, H., Sambruna, R., Chartas, G., Garmire, G. P., \& Nousek, J. A. 2000, ApJ, 535, L17

Komossa, S., \& Mathur, S. 2001, A\&A, 374, 914

Komossa, S., \& Meerschweinchen, J. 2000, A\&A, 354, 411

Korista, K., Ferland, G., \& Baldwin, J. 1997, ApJ, 487, 555

Krolik, J. H., \& Kriss, G. A. 1995, ApJ, 447, 512

Krolik, J. H., McKee, C. F., \& Tarter, C. B. 1981, ApJ, 249, 422

Krongold, Y., Nicastro, F., Brickhouse, N. S., Elvis, M., Liedahl, D. A., \& Mathur, S. 2003, ApJ, 597, 832

Krongold, Y., Nicastro, F., Brickhouse, N. S., Elvis, M., \& Mathur, S. 2005a, ApJ, 622, 842

Krongold, Y., Nicastro, F., Elvis, M., Brickhouse, N. S., Mathur, S., \& Zezas, A. 2005b, ApJ, 620, 165

Lee, J. C., et al. 2013, MNRAS, 803

Lobban, A. P., Reeves, J. N., Miller, L., Turner, T. J., Braito, V., Kraemer, S. B., \& Crenshaw, D. M. 2011, MNRAS, 414, 1965

Longinotti, A. L., Cappi, M., Nandra, K., Dadina, M., \& Pellegrini, S. 2003, A\&A, 410, 471

Longinotti, A. L., Nucita, A., Santos-Lleo, M., \& Guainazzi, M. 2008, A\&A, 484, 311

Makishima, K., Maejima, Y., Mitsuda, K., Bradt, H. V., Remillard, R. A., Tuohy, I. R., Hoshi, R., \& Nakagawa, M. 1986, ApJ, 308, 635

Matt, G., et al. 2011, A\&A, 533, A1

Mehdipour, M., Branduardi-Raymont, G., \& Page, M. J. 2010, A\&A, 514, A100

-. 2012, A\&A, 542, A30

Nandra, K., \& Pounds, K. A. 1994, MNRAS, 268, 405

Netzer, H. 1993, ApJ, 411, 594

一. 1996, ApJ, 473, 781

Netzer, H., et al. 2003, ApJ, 599, 933

Nicastro, F., Fiore, F., \& Matt, G. 1999, ApJ, 517, 108

Nucita, A. A., Guainazzi, M., Longinotti, A. L., Santos-Lleo, M., Maruccia, Y., \& Bianchi, S. 2010, A\&A, 515, A47+ 
TABLE 1

The Best fit model parameters for CLOUDY models using Kirk Korista and the Realistic ionizing Continua.

\begin{tabular}{|c|c|c|c|}
\hline $\begin{array}{l}\text { Model } \\
\text { components }\end{array}$ & parameters & $\begin{array}{l}\text { Kirk Korista }{ }^{\mathrm{a}, \mathrm{c}} \\
\text { WA model }\end{array}$ & $\begin{array}{l}\text { Appropriate }{ }^{\mathrm{b}, \mathrm{c}} \\
\text { WA model }\end{array}$ \\
\hline wabs & $\begin{array}{l}\mathrm{N}_{\mathrm{H}} \times 10^{20}\left(\mathrm{~cm}^{-2}\right) \\
\text { (fixed) }\end{array}$ & $1.1(f)$ & $1.1(f)$ \\
\hline Warm absorber & $\log \left(N_{\mathrm{H}}^{\mathrm{WA}} / \mathrm{cm}^{-2}\right)$ & $21.58_{-0.07}^{+0.10}$ & $21.33_{-0.04}^{+0.09}$ \\
\hline \multirow[t]{2}{*}{ (CLOUDY) } & $\log \left(\xi / \mathrm{erg} \mathrm{cm} \mathrm{s}^{-1}\right)$ & $1.50_{-0.07}^{+0.08}$ & $1.75_{-0.09}^{+0.13}$ \\
\hline & outflow-velocity d & $960_{-450}^{+480}$ & $960_{-450}^{+480}$ \\
\hline Warm absorber & $\log \left(N_{\mathrm{H}}^{\mathrm{WA}} / \mathrm{cm}^{-2}\right)$ & $21.31_{-0.16}^{+0.30}$ & $21.36_{-0.25}^{+0.23}$ \\
\hline \multirow[t]{2}{*}{ (CLOUDY) } & $\log \left(\xi / \mathrm{erg} \mathrm{cm} \mathrm{s}^{-1}\right)$ & $2.25_{-0.09}^{+0.13}$ & $3.24_{-0.16}^{+0.22}$ \\
\hline & outflow-velocity d & $1170_{-600}^{+900}$ & $1200_{-600}^{+900}$ \\
\hline bbody & $\begin{array}{l}\mathrm{kT}_{\mathrm{BB}}(\mathrm{eV}) \\
\text { norm }\end{array}$ & $\begin{array}{l}85_{-2}^{+1} \\
(12 \pm 2)^{\mathrm{e}}\end{array}$ & $\begin{array}{l}87_{-2}^{+1} \\
(12 \pm 2)^{\mathrm{e}}\end{array}$ \\
\hline $\begin{array}{l}\text { nthcomp } \\
\text { (powerlaw) }\end{array}$ & $\begin{array}{l}\Gamma \\
\text { norm }\end{array}$ & $\begin{array}{l}2.00 \pm 0.03 \\
(90 \pm 20)^{\mathrm{e}}\end{array}$ & $\begin{array}{l}2.00 \pm 0.02 \\
(90 \pm 20)^{\mathrm{e}}\end{array}$ \\
\hline Gaussian & $\begin{array}{l}\text { norm } \\
\text { Line E (rest) keV } \\
\sigma(\mathrm{eV})\end{array}$ & $\begin{array}{l}(0.16 \pm 0.13)^{\mathrm{c}} \\
6.4 \pm 0.007 \\
0.001 \mathrm{keV}(\mathrm{f})\end{array}$ & $\begin{array}{l}(0.16 \pm 1.3)^{\mathrm{e}} \\
6.4 \pm 0.005 \\
0.001 \mathrm{keV}(\mathrm{f})\end{array}$ \\
\hline edge & $\begin{array}{l}\text { Energy }(\mathrm{keV}) \\
\tau\end{array}$ & $\begin{array}{l}7.48 \pm 0.12 \\
0.44 \pm 0.22\end{array}$ & $\begin{array}{l}7.48 \pm 0.12 \\
0.40 \pm 0.22\end{array}$ \\
\hline $\mathrm{C} / \mathrm{dof}$ & & $5940 / 5139$ & $5935 / 5139$ \\
\hline $\begin{array}{l}\chi^{2} / \text { dof } \\
\text { (EPIC-pn fit only) }\end{array}$ & & $213 / 181$ & $214 / 181$ \\
\hline
\end{tabular}

${ }^{a}$ WA table model generated using Kirk Korista continuum.

${ }^{b}$ WA table model generated using the appropriate continuum with the SE modeled with a bbody. See Sec. 3.6

${ }^{\mathrm{c}}(f)$ signifies frozen parameters.

${ }^{\mathrm{d}}$ The outflow velocity of the WA with respect to systemic velocity expressed in $\mathrm{km} \mathrm{s}^{-1}$.

eThese quantities are in the units of $10^{-5}$. 
TABLE 2

THE BEST FIT WARM ABSORBER MODEL PARAMETERS WITH EITHER THE UV OR THE SOFT-EXCESS PART OR BOTH THE PARTS OF THE REALISTIC CONTINUUM SWITCHED OFF.

\begin{tabular}{lllll}
\hline \hline $\begin{array}{l}\text { WA } \\
\text { Component }\end{array}$ & Parameters & Model $1^{\mathrm{a}}$ & Model $2^{\mathrm{a}}$ & Model $3^{\mathrm{a}}$ \\
\hline & & & \\
\hline & $\log \left(N_{\mathrm{H}}^{\mathrm{WA}} / \mathrm{cm}^{-2}\right)$ & $21.33_{-0.05}^{+0.09}$ & $21.36_{-0.12}^{+0.12}$ & $21.38_{-0.12}^{+0.12}$ \\
& $\log \left(\xi / \mathrm{erg} \mathrm{cm} \mathrm{s}^{-1}\right)$ & $0.99_{-0.06}^{+0.08}$ & $2.29_{-0.09}^{+0.09}$ & $1.30_{-0.1}^{+0.1}$ \\
& & & & \\
& $\log \left(N_{\mathrm{H}}^{\mathrm{WA}} / \mathrm{cm}^{-2}\right)$ & $21.49_{-0.31}^{+0.11}$ & $21.44_{-0.40}^{+0.12}$ & $21.44_{-0.40}^{+0.12}$ \\
& $\log \left(\xi / \mathrm{erg} \mathrm{cm} \mathrm{s}^{-1}\right)$ & $2.50_{-0.07}^{+0.12}$ & $3.30_{-0.09}^{+0.14}$ & $2.30_{-0.09}^{+0.14}$ \\
& & & & \\
\hline
\end{tabular}

$\mathrm{C} / \mathrm{dof}$

$5935 / 5139 \quad 5932 / 5139 \quad 5932 / 5139$

${ }^{\text {a Model }} 1$ stands for the BBB switched off from the NLSy1 SED as described in section 2 .

Model 2 stands for SE swiched off

Model 3 stands for the BBB as well as the SE switched off.

TABLE 3

THE BEST FIT WARM ABSORBER MODEL PARAMETERS WHEN WE USE REALISTIC IONIZING CONTINUA TO GENERATE CLOUDY WARM ABSORBER TABLE MODELS.

\begin{tabular}{lllll}
\hline \hline $\begin{array}{l}\text { WA } \\
\text { component }-\end{array}$ & Parameters & $\begin{array}{l}\text { Model 1 } \\
(\text { bbody })\end{array}$ & $\begin{array}{l}\text { Model 2 } \\
(\text { reflionx })\end{array}$ & $\begin{array}{l}\text { Model 3 } \\
\text { (optxagnf })\end{array}$ \\
\hline & & & & \\
1. & $\log \left(N_{\mathrm{H}}^{\mathrm{WA}} / \mathrm{cm}^{-2}\right)$ & $21.33_{-0.04}^{+0.09}$ & $21.40_{+0.04}^{-0.12}$ & $21.36_{+0.12}^{-0.09}$ \\
& $\log \left(\xi / \mathrm{erg} \mathrm{cm} \mathrm{s}^{-1}\right)$ & $1.75_{-0.09}^{+0.13}$ & $1.88_{-0.12}^{+0.16}$ & $1.95_{-0.08}^{+0.09}$ \\
& & & & \\
& $\log \left(N_{\mathrm{H}}^{\mathrm{WA}} / \mathrm{cm}^{-2}\right)$ & $21.36_{-0.25}^{+0.23}$ & $21.91_{-0.25}^{+0.13}$ & $21.59_{-0.15}^{+0.25}$ \\
& & & \\
& $\log \left(\xi / \mathrm{erg} \mathrm{cm} \mathrm{s}^{-1}\right)$ & $3.25_{-0.16}^{+0.22}$ & $3.55_{-0.25}^{+0.11}$ & $3.52_{-0.15}^{+0.08}$ \\
& & & & \\
\hline
\end{tabular}

C/dof

$5935 / 51395927 / 5139 \quad 5930 / 5139$

a Models 1, 2 and 3 stands for cases when SE is described by bbody, reflionx and optxagnf. See Sec. 3.7 for details.
Piconcelli, E., Jimenez-Bailón, E., Guainazzi, M., Schartel, N., Rodríguez-Pascual, P. M., \& Santos-Lleó, M. 2005, A\&A, 432, 15

Porter, R. L., Ferland, G. J., Kraemer, S. B., Armentrout, B. K., Arnaud, K. A., \& Turner, T. J. 2006, PASP, 118, 920

Reynolds, C. S. 1997, in Astronomical Society of the Pacific Conference Series, Vol. 128, Mass Ejection from Active Galactic Nuclei, ed. N. Arav, I. Shlosman, \& R. J. Weymann, 173-+

Reynolds, C. S., \& Fabian, A. C. 1995, MNRAS, 273, 1167

Ricci, C., Beckmann, V., Audard, M., \& Courvoisier, T. J.-L. 2010, A\&A, 518, A47

Ross, R. R., \& Fabian, A. C. 2005, MNRAS, 358, 211

Sako, M., et al. 2001, A\&A, 365, L168

Schlafly, E. F., \& Finkbeiner, D. P. 2011, ApJ, 737, 103

Schlegel, D. J., Finkbeiner, D. P., \& Davis, M. 1998, ApJ, 500, 525

Shakura, N. I., \& Sunyaev, R. A. 1973, A\&A, 24, 337

Smith, R. A. N., Page, M. J., \& Branduardi-Raymont, G. 2008, A\&A, 490, 103

Steenbrugge, K. C., Kaastra, J. S., de Vries, C. P., \& Edelson, R. 2003, A\&A, 402, 477

Tarter, C. B., Tucker, W. H., \& Salpeter, E. E. 1969, ApJ, 156, 943

Winter, L. M., Veilleux, S., McKernan, B., \& Kallman, T. R. 2012, ApJ, 745, 107

Yaqoob, T., McKernan, B., Kraemer, S. B., Crenshaw, D. M., Gabel, J. R., George, I. M., \& Turner, T. J. 2003, ApJ, 582, 105 
TABLE 4

The best fit parameters for the Simultaneous fit of the EPIC-Pn and RGS Data When the Soft-excess Was modeled using PHYSICAL MODELS.

\begin{tabular}{|c|c|c|c|}
\hline $\begin{array}{l}\text { Model } \\
\text { components }\end{array}$ & paramters & Model $1^{\mathrm{a}, \mathrm{b}}$ & Model $2^{\mathrm{a}, \mathrm{b}}$ \\
\hline wabs & $\begin{array}{l}\mathrm{N}_{\mathrm{H}}\left(\mathrm{cm}^{-2}\right) \times 10^{20} \\
\text { (fixed) }\end{array}$ & $1.1(f)$ & $1.1(f)$ \\
\hline WA1 and WA2 & Values quoted in Table 3 & & \\
\hline powerlaw & $\begin{array}{l}\Gamma \\
\text { norm }\end{array}$ & $\begin{array}{l}2.04 \pm 0.03 \\
(5.9 \pm 2) \times 10^{-4}\end{array}$ & - \\
\hline Reflionx-1 & $\begin{array}{l}\mathrm{Fe} / \text { solar } \\
\Gamma \\
\xi \\
\text { norm }\end{array}$ & $\begin{array}{l}10_{-0.75}^{+0.0} \\
2.04 \\
199_{-14}^{+3} \\
(1.43 \pm 0.19) \times 10^{-6}\end{array}$ & $\begin{array}{l}- \\
- \\
-\end{array}$ \\
\hline Kdblur1 & $\begin{array}{l}\text { index } \\
\mathrm{R}_{\text {in }}\left(\mathrm{r}_{\mathrm{g}}\right) \\
\mathrm{R}_{\text {out }}\left(\mathrm{r}_{\mathrm{g}}\right) \\
\text { inclination(degrees) }\end{array}$ & $\begin{array}{l}9.58_{-0.41}^{+0.50} \\
1.23_{-0.00}^{+0.07} \\
13.2_{-0.2}^{+0.5} \\
37_{-6}^{+3}\end{array}$ & $\begin{array}{l}- \\
- \\
-\end{array}$ \\
\hline Reflionx-2 & $\begin{array}{l}\mathrm{Fe} / \text { solar } \\
\Gamma \\
\xi \\
\text { norm }\end{array}$ & $\begin{array}{l}10(\text { tied }) \\
2.04 \\
60_{-8}^{+21} \\
\left(4.0_{-1.0}^{+0.5}\right) \times 10^{-7}\end{array}$ & $\begin{array}{l}- \\
- \\
-\end{array}$ \\
\hline Kdblur2 & $\begin{array}{l}\text { index } \\
\mathrm{R}_{\text {in }}\left(\mathrm{r}_{\mathrm{g}}\right) \\
\mathrm{R}_{\text {out }}\left(\mathrm{r}_{\mathrm{g}}\right) \\
\text { inclination(degrees) }\end{array}$ & $\begin{array}{l}3.55_{-1.5}^{+0.5} \\
13.2(\text { tied }) \\
400(f) \\
37(\text { tied })\end{array}$ & - \\
\hline Reflionx-3 & $\begin{array}{l}\mathrm{Fe} / \text { solar } \\
\Gamma \\
\xi \\
\text { norm }\end{array}$ & $\begin{array}{l}0.75_{-0.18}^{+0.41} \\
2.04 \\
139_{-28}^{+76} \\
7.26_{2.20}^{+4.20} \times 10^{-8}\end{array}$ & $\begin{array}{l}- \\
- \\
-\end{array}$ \\
\hline optxagnf & $\begin{array}{l}\text { norm } \\
\log \left(\mathrm{L} / \mathrm{L}_{\mathrm{EDD}}\right) \\
\mathrm{kT}_{\mathrm{e}}(\mathrm{keV}) \\
\tau \\
\Gamma \\
\mathrm{f}_{\mathrm{pl}} \\
\mathrm{r}_{\text {cor }}\left(\mathrm{r}_{\mathrm{g}}\right)\end{array}$ & $\begin{array}{l}- \\
- \\
- \\
- \\
-\end{array}$ & $\begin{array}{l}1(f) \\
-0.755_{-0.004}^{+0.003} \\
0.101_{-0.02}^{+0.04} \\
68_{-6}^{+5} \\
1.99 \pm 0.02 \\
0.51 \pm 0.02 \\
10 \pm 2\end{array}$ \\
\hline Gaussian & $\begin{array}{l}\text { norm } \\
\text { Line } \mathrm{E} \text { (rest) } \mathrm{keV} \\
\sigma(\mathrm{eV})\end{array}$ & - & $\begin{array}{l}6.7_{+2.0}^{-2.2} \times 10^{-7} \\
6.4 \pm 0.005 \\
0.001 \mathrm{keV}(\mathrm{f})\end{array}$ \\
\hline edge & $\begin{array}{l}\text { Energy }(\mathrm{keV}) \\
\tau\end{array}$ & - & $\begin{array}{l}7.48 \pm 0.20 \\
0.46 \pm 0.22\end{array}$ \\
\hline $\mathrm{C} / \mathrm{dof}$ & & $5927 / 5139$ & $5930 / 5139$ \\
\hline $\begin{array}{l}\chi^{2} / \text { dof } \\
\text { (EPIC-pn fit only) }\end{array}$ & & $201 / 171$ & $223 / 176$ \\
\hline
\end{tabular}

${ }^{a}$ Model 1=wabs*WA1*WA2*edge*(powerlaw+kdblur(Reflion)+kdblur2(Reflion2)),

Model 2 =wabs*WA $1 *$ WA $2 *$ edge* (optxagnf+Gaussian)

${ }^{\mathrm{b}}(f)$ stands for frozen parameters 